\title{
Kongenitale Enteropathie und Darmtransplantation
}

\author{
Olivier Goulet
}

Referenzzentrum für seltene Erkrankungen des Verdauungstrakts, Abteilung für pädiatische Gastroenterologie, Hepatologie und Ernährung, Hôpital Necker - Enfants Malades, Paris, Frankreich

\section{Schlüsselwörter \\ Darmversagen • Citrullin • Kongenitale Enteropathie • Parenterale Ernährung • Lebererkrankung • Darmtransplantation · Lebertransplantation • Parenterale Ernährung zu Hause}

\section{Zusammenfassung}

Bei Darmversagen muss der Patient parenteral ernährt werden. Zu den Ursachen eines schweren protrahierten Darmversagens zählen das Kurzdarmsyndrom, schwere Motilitätsstörungen (eine totale oder segmentale Aganglionose oder eine chronische intestinale Pseudoobstruktion) sowie kongenitale Störungen bei der Bildung der Enterozyten. Bei Patienten mit Darmversagen können schwere Lebererkrankungen auftreten, die entweder eine Folge der zugrundeliegenden Erkrankung des Verdauungssystems sind oder sich infolge einer nicht richtig auf die Bedürfnisse des Patienten angepassten parenteralen Ernährung (PE) ergeben. Kathetersepsis oder eine ausgedehnte vaskuläre Thrombose behindern möglicherweise die Weiterführung einer verträglichen und wirksamen PE. Zum Management von Patienten mit Darmversagen muss die Erkrankung daher frühzeitig erkannt und bezüglich des Risikos der Irreversibilität untersucht werden. Nach wie vor entscheidend ist der richtige Zeitpunkt für die Indikationsstellung zur Darmtransplantation. Das Management von Patienten mit Darmversagen sollte daher Therapien für jedes Stadium dieser Erkrankung umfassen, die auf einem multidisziplinären Ansatz beruhen und in Zentren durchgeführt werden, die eine Abteilung für pädiatrische Gastroenterologie sowie für pädiatrische Chirurgie haben, über Expertenwissen im Bereich der PE verfügen und ein Programm für die PE zu Hause sowie für die Leber-DarmTransplantation bieten. Darmversagen ist eine Erkrankung, die mit schwerer intestinaler Malabsorption einhergeht und PE erfordert. Kongenitale Enteropathien, welche die Darmschleimhaut schädigen, führen zu irreversiblem Darmversagen. Die parenterale Dauerernährung birgt ein hohes Komplikationsrisiko und steigert daher möglicherweise die Notwendigkeit einer Darmtransplantation.

Copyright (C) 2006 Nestec Ltd., Vevey/S. Karger AG, Basel

\section{Definition des Darmversagens}

«Darmversagen» wird wie folgt definiert: Eine kritische Reduktion der intestinalen Resorptionsfläche in einem solchen Ausmass, dass eine ausreichende Verdauung und Resorption zur Deckung des Nährstoff- und Flüssigkeitsbedarfs des Körpers und damit die Erhaltung der Gesundheit bei Erwachsenen und das Wachstum bei Kindern nicht mehr gewährleistet ist. Diese Definition wird jedoch nicht von allen akzeptiert. Manche meinen, es sei besser bei Patienten mit Kurzdarmsyndrom das Darmversagen anhand der über die fäkale Exkretion entstehenden Energieverluste und nicht anhand der Restdarmlänge zu definieren [1]. Bei einem anderen Ansatz definiert man den Grad des intestinalen Versagens anhand der zum Erhalt der Gesundheit bei Erwachsenen

\section{KARGER}

Fax +4161306 1234

E-Mail karger@karger.ch

www.karger.com
C) 2006 Nestec Ltd. Vevey/S. Karger AG, Basel 0250-9652/06/0641-0025\$23.50/0

Online-Zugriff auf: www.karger.com/and
Olivier Goulet, Reference Center for Rare Digestive Diseases

Department of Pediatric Gastroenterology, Hepatology and Nutrition

Hôpital Necker - Enfants Malades, 149 Rue de Sèvres

FR-75743 Paris Cedex 15 (France)

Tel. +331444944 11, Fax +331444925 01, E-Mail olivier.goulet@nck.ap-hop-paris.fr 
bzw. des Wachstums bei Kindern erforderlichen Menge an Sondennahrung zur parenteralen Ernährung (PE). Vor kurzem wurde, abgesehen von den anatomischen und ernährungsbedingten Kriterien zur Definition des Darmversagens, auf einen biologischen Marker hingewiesen, mit dem sich die intestinale Resorptionsfläche des Darms bestimmen lässt [2]. Citrullin ist eine nicht-essenzielle Aminosäure, die hauptsächlich von den Enterozyten gebildet wird [3]. Die Citrullinkonzentration im Blutplasma lässt sich leicht bestimmen und spiegelt bei Patienten mit Kurzdarmsyndrom, Patienten mit verschiedengradiger Zottenatrophie und Patienten mit Transplantatabstossung und Zottenatrophie den Verlust der intestinalen Resorptionsfläche des Darms wider [4]. In einer unlängst durchgeführten Studie, in der pädiatrischen Patienten mit Kurzdarmsyndrom rekombinantes, humanes Wachstumshormon verabreicht wurde, konnte nach der Behandlung ein signifikanter Anstieg der Citrullinspiegel im Blutplasma festgestellt werden [5]. Gegenwärtig wird das Darmversagen jedoch weiterhin wie folgt definiert: Die Erhaltung des Wachstums bei Kindern erweist sich ohne parenterale Dauerernährung als unmöglich.

\section{Ursachen des Darmversagens}

Der Verlauf des Darmversagens bei Kindern ist unterschiedlich und wird weitgehend von der zugrundeliegenden Erkrankung, die für den Verlust der Darmfunktion verantwortlich ist, beeinflusst. Darmversagen tritt am häufigsten sekundär infolge eines gastrointestinalen Infekts auf. Man geht jedoch davon aus, dass Kinder, die unter einem solchen akuten Darmversagen leiden, bei entsprechender Behandlung vollständig genesen. Nichtsdestotrotz zählt die protrahierte, nach Enteritis auftretende Diarrhoe in Entwicklungsländern nach wie vor zu den Hauptursachen von Darmversagen [6]. In den Industrieländern sind die meisten Fälle von Darmversagen auf schwere und chronische Erkrankungen (kongenitale oder Early-Onset-Fälle) zurückzuführen, die eine PE von längerer oder unbegrenzter Dauer notwendig machen. Eine der ersten in der Vergangenheit anerkannten Ursachen des Darmversagens war das Kurzdarmsyndrom [7]. Mit zunehmendem und erfolgreichem Einsatz der parenteralen Dauerernährung in den letzten dreissig Jahren, zeichneten sich jedoch diverse andere Ursachen des Darmversagens ab, wie z.B. schwere ausgedehnte Motilitätsstörungen, u.a. segmentale Aganglionose, chronische intestinale Pseudoobstruktion und kongenitale Erkrankungen der Enterozytenbildung [8].

\section{Die kongenitale Enteropathie als Ursache des Darmversagens}

Ausgehend von immunhistologischen Kriterien, welche die Rolle von aktivierten T-Zellen in der Darmschleimhaut betonen, schlug man vor, die intraktable Diarrhoe im Säuglingsalter anhand des Grads der Zottenatrophie zu klassifizieren [9]. Im Rahmen einer multizentrischen Studie der ESPGHAN wurden Fälle von intraktabler Diarrhoe im Säuglingsalter gesammelt, die im Lichtmikroskop exakt definierte Merkmale aufwiesen und somit eine Kategorisierung der verschiedenen Arten der intraktablen Diarrhoe im Säuglingsalter ermöglicht. Kennzeichnend für die erste Gruppe war eine mononukleäre Zellinfiltration der Lamina propria, bei der man einen Zusammenhang mit aktivierten T-Zellen vermutete. Das zweite histologische Muster umfasste eine schwere frühkindliche Diarrhoe mit Zottenatrophie, jedoch ohne mononukleäre Zellinfiltration der Lamina propria und mit spezifischen histologischen Befunden im Epithel. Bisher wurden mehrere Arten primärer Epithelveränderungen festgestellt, welche die intraktable Diarrhoe im Säuglingsalter hervorrufen. Die Mikrovillusatrophie (MVA) bzw. die Enteropathie mit Einschluss von Mikrovilli war die erste dieser Veränderungen, die beschrieben wurde. Vor nicht allzu langer Zeit wurde diese Beschreibung um die Tufting-Enteropathie bzw. intestinale Epitheldysplasie (IED) ergänzt [11]. Manche SGA-Kinder werden mit phänotypischen Anomalien vorstellig, die der protrahierten, therapieresistenten Diarrhoe im Säuglingsalter entsprechen [12].

\section{Mikrovillusatrophie}

Im Jahr 1978 berichteten Davidson et al. [13] über 5 Säuglinge mit schwerer persistierender Diarrhoe, die im Neugeborenenalter einsetzte. Lichtmikroskopisch zeigte sich eine Zottenatrophie mit Kryptenhypoplasie. Eine elektronenmikroskopische Untersuchung von Darmbiopsien, die drei dieser Patienten entnommen worden waren, zeigte starke Veränderungen des Bürstensaums und vermehrt liposomenartige Gebilde. In einer der Biopsien waren intrazytoplasmatische Zysten mit Bürstensaumstruktur festzustellen. Ferner wurde über Kinder mit charakteristischen Zytoplasmaeinschlüssen der Bürstensaummembran berichtet [14]. Ausgehend von diesen klinischen und histologischen Beschreibungen wurde die MVA als eigenständige Krankheit innerhalb des Syndroms der intraktablen Diarrhoe im Säuglingsalter nachgewiesen. Bei der MVA handelt es sich um eine kongenitale Erkrankung der intestinalen Epithelzel- 
Abb. 1. PAS (periodic acid-Schiff)-Färbung. A Normale Mucosa, normale PASFärbung des Bürstensaums. B Abnorme Ansammlung von PAS-positivem Material im apikalen Zytoplasma der Epithelzellen bei der Enteropathie mit Einschluss von Mikrovilli.
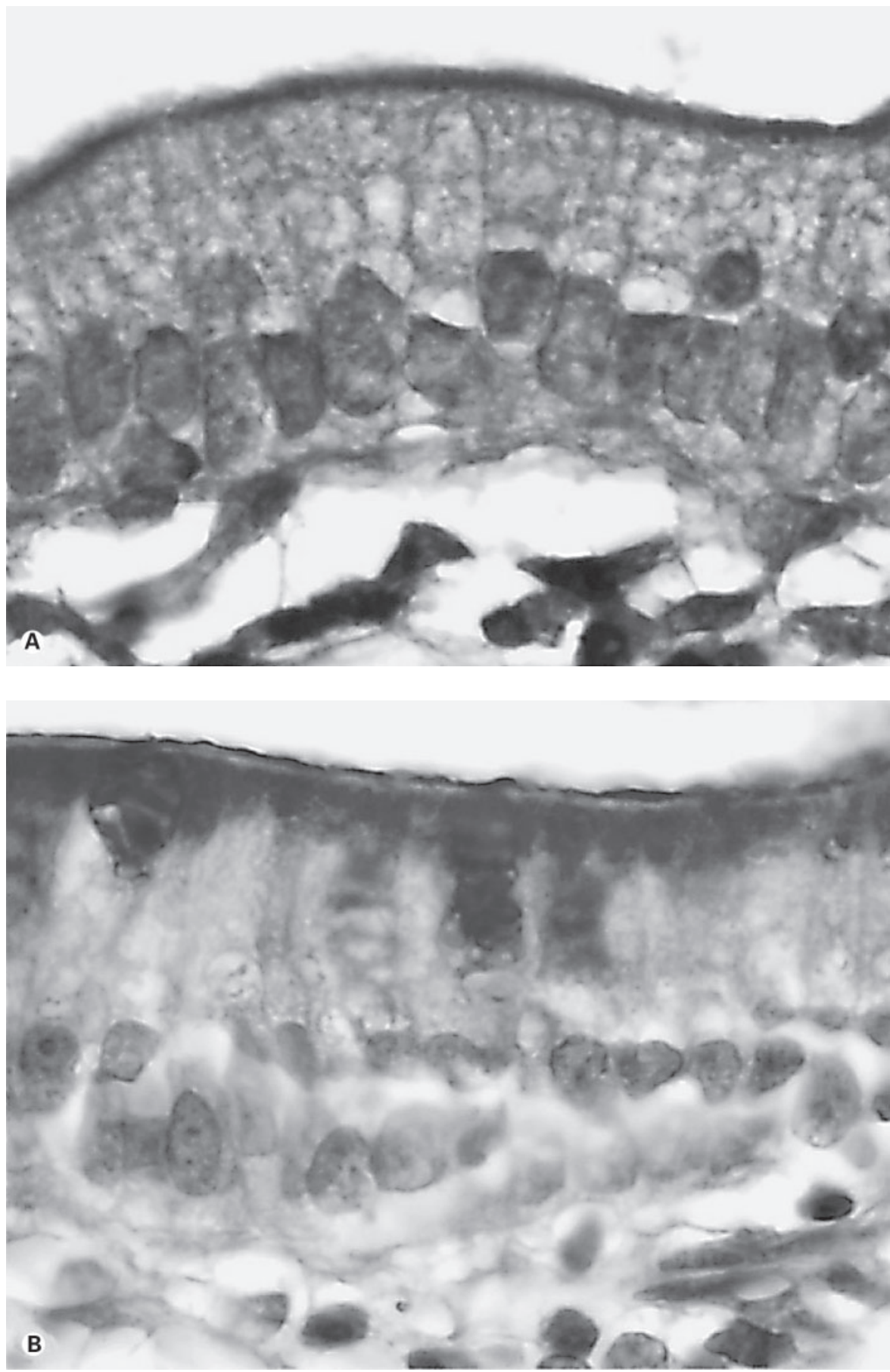

len, die sich anfänglich als therapieresistente Neugeborenendiarrhoe äussert.

\section{Klinisches Bild}

Bei den betroffenen Säuglingen tritt in der Regel in den ersten Tagen nach der Geburt eine starke, wässrige Diarrhoe auf. Die tägliche Stuhlmenge kann bis zu 250-
$300 \mathrm{ml} / \mathrm{kg}$ Körpergewicht betragen und die Elektrolytkonzentrationen in den Faeces entsprechen den Elektrolytkonzentrationen der Dünndarmflüssigkeit. Diese Erkrankung ist besonders lebensbedrohlich, da massive Diarrhoe schnell Dehydration und ein Elektrolytungleichgewicht verursachen kann, die in der Folge innerhalb weniger Stunden zur metabolischen Dekompensati- 
Abb. 2. Enteropathie mit Einschluss von Mikrovilli. Elektronenmikroskopische Aufnahme einer Biopsieprobe aus dem Jejunum. Der Bürstensaum fehlt fast völlig. Das Zytoplasma enthält einen Einschluss von Mikrovilli.

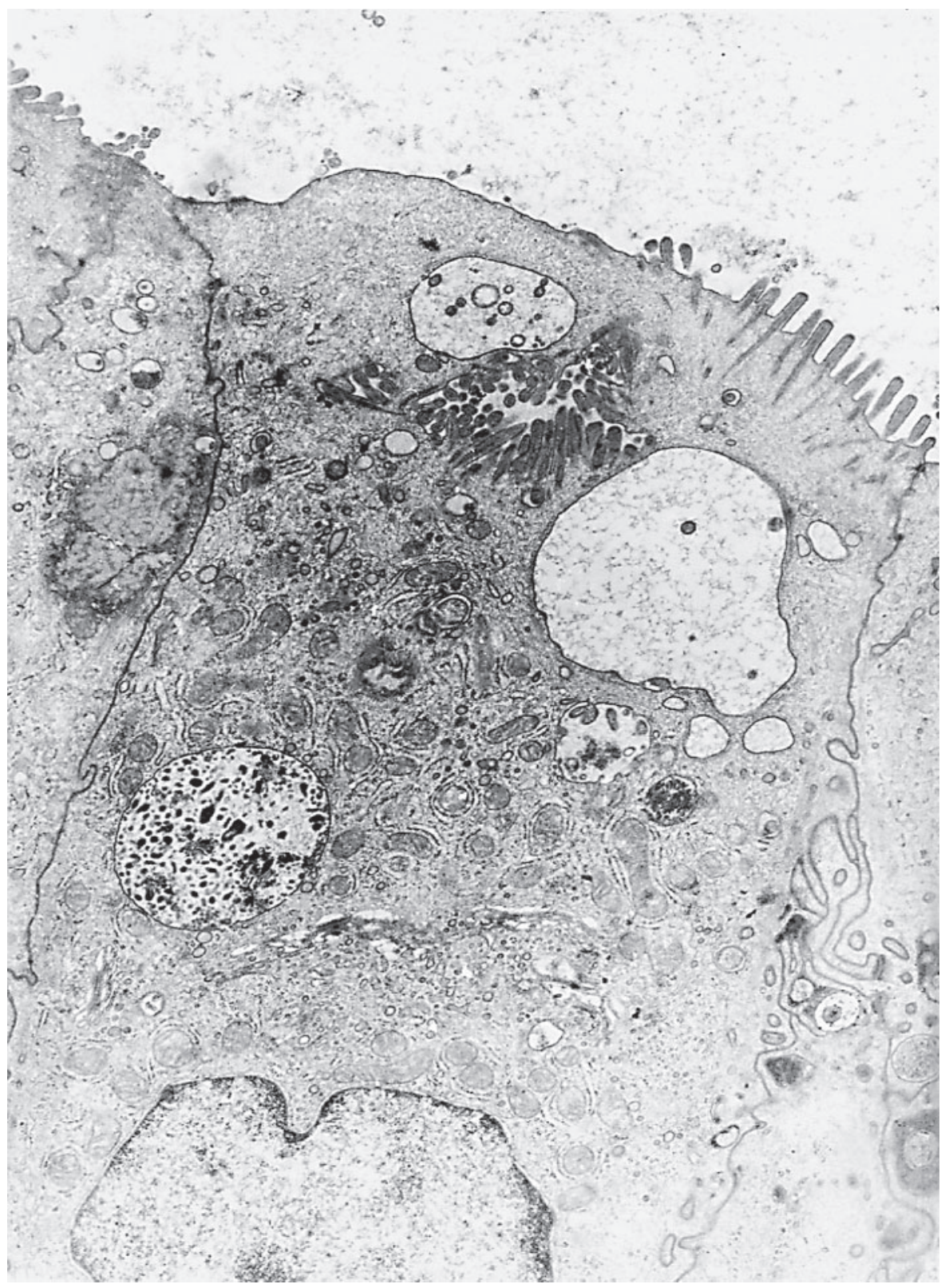

on führen. Die starken, wässrigen Durchfälle persistieren, auch wenn der Darm zur Ruhe kommt. Die Differenzialdiagnose schliesst möglicherweise die kongenitale Chloriddiarrhoe und die Diarrhoe bei Natrium-Malabsorption ein, die anhand einer Bestimmung der Elektrolytgehalte in Blut und Faeces jedoch leicht von der MVA zu unterscheiden sind [15-16]. Im Gegensatz dazu kann sich die MVA auch äusserst atypisch präsentieren, mit einem vollen und aufgeblähten Dünn- und Dickdarm, ähnlich wie bei einer intestinalen Pseudoobstruktion. In den meisten MVA-Fällen setzt die Diarrhoe in den ersten
Lebenstagen ein (Early-Onset-Fälle oder kongenitale Form). Bei einigen Patienten jedoch, kann die Diarrhoe später auftreten (während des ersten Lebensmonats) oder weniger stark ausgeprägt sein (Late-Onset-Fälle).

\section{Histopathologische Analysen}

Mittels lichtmikroskopischer Untersuchung von Biopsieproben aus dem Duodenum oder Jejunum ist die Diagnose einfach zu stellen. Die Hematoxylin-Eosin-Färbung zeigt eine abgeflachte Darmschleimhaut mit hypoplastischer Villusatrophie. 
Äusserst charakteristisch für diese Erkrankung ist die Ansammlung von PAS-(periodic acid-Schiff)-positiven Sekretgranula im apikalen Zytoplasma der Enterozyten $[17,18]$ (Abb. 1). Auf ultrastruktureller Ebene sind nur vereinzelte bzw. gar keine Mikrovilli sowie zytoplasmatische Einschlüsse von Mikrovilli oder Enterozyten zu sehen. Hierbei handelt es sich um anatomische Veränderungen, die für diese Krankheitseinheit typisch sind [19]. Vor kurzem wurde ein neues, wertvolles Tool für die Diagnose der MVA mittels lichtmikroskopischer Untersuchung vorgeschlagen [20,21]. Die neutrale Aminopeptidase CD-10 ist membranassoziiert und bei einem gesunden Dünndarm ergibt sich nachweislich eine lineare Färbung des Bürstensaums. Im Gegensatz zu dieser Oberflächenfärbung beim gesunden Darm und bei diversen Darmerkrankungen (Zöliakie, autoimmune Enteropathie und Allergie) ist bei allen MVA-Fällen bei den oberflächlichen Enterozyten eine ausgeprägte intrazytoplasmatische CD-10-Immunreaktivität festzustellen [20]. Ähnliche Ergebnisse wurden mit PAS, polyklonalem CEA (karzinoembryonalem Antigen) und alkalischer Phosphatase erzielt, drei Färbemethoden, die bei MVA bekanntermassen eine zytoplasmatische Färbung der oberflächlichen Enterozyten erkennen lassen [22]. Im Elektronenmikroskop erkennt man, dass die Mikrovilli der Oberflächenepithelzellen fehlen bzw. stark verändert sind, und es lassen sich zahlreiche bläschenartige Gebilde verschiedener Grösse sowie charakteristische Einschlüsse mit Mikrovilli feststellen (Abb. 2). Die Kryptenzellen sind morphologisch fast normal. Sie enthalten keine erhöhte Anzahl apikaler Vesikel und bläschenartiger Gebilde. Auch im Dickdarmepithel finden sich MikrovilliEinschlüsse und vermehrt Sekretgranula. Dies ist besonders wichtig, da es insbesondere bei sehr kleinen Säuglingen einfacher ist, Biopsien aus dem Dickdarmgewebe zu entnehmen.

\section{Pathophysiologie und Übertragungsart}

Auch ein Defekt im Membrantrafficking unreifer bzw. differenzierender Enterozyten wurde als ein möglicher Mechanismus der Ätiopathogenese der MVA erörtert [19, 22]. Vollständiges Darmversagen ist eine direkte funktionelle Folge dieses Membrandefekts. Man vermutet, dass diese Krankheit mit einer Störung des Zytoskeletts der Enterozyten zusammenhängt. Das Zytoskelett produziert eine abnorme Anordnung der Mikrovilli. Es gibt einen Bericht über eine hypothetische Variante der MVA, nämlich die Mikrovillusdystrophie [22]. Die der MVA zugrunde liegende Pathogenese ist jedoch nach wie vor unklar. Eine Analyse des Turnovers von Sucrase-Iso- maltase, einem repräsentativen Protein des Bürstensaums, belegte eindeutig, dass die direkte und indirekte konstitutive Exozytose bei der MVA intakt ist [19]. Da die Mikrovilli sowohl bei der kongenitalen MVA als auch bei Late-Onset-Fällen in den Bereichen, in denen sich die Sekretgranula zuerst ansammeln, normal zu sein scheinen, kann ein Defekt in der Biosynthese der Mikrovilli als Ursache der Erkrankung wahrscheinlich ausgeschlossen werden. Phillips et al. [23] untersuchten die Sekretgranula und wiesen vor kurzem darauf hin, dass eine Störung der Exozytose von Glykocalyx, die möglicherweise eine Störung der normalen Zellfunktionen verursacht, beteiligt sein könnte.

Die Krankheit scheint in Anbetracht der Anzahl an Fällen, bei denen Geschwister ebenfalls unter der Krankheit leiden, und in Anbetracht der Konsanguinitätsrate bei Patienten autosomal rezessiv vererbt zu werden [24]. Ein Kandidatengen wurde bislang nicht entdeckt, aber es wurde ein Fall gemeldet, bei dem bei einem Mädchen mit autosomal dominanter Hypochondroplasie eine MVA diagnostiziert wurde [25]. Der Gendefekt, der dieser Hypochondroplasie zugrunde liegt, wurde vor kurzem auf der Chromosomenregion 4p16.3 lokalisiert. Diese könnte unter Umständen zur Aufklärung der genetischen Grundlage der MVA beitragen.

\section{Langfristiges Outcome}

Die grösste, an 23 MVA-Patienten durchgeführte Multizenter-Studie [14] ergab eine extrem reduzierte Lebenserwartung mit einer Ein-Jahres-Überlebensrate von unter $25 \%$. Die meisten Kinder starben an septischen Komplikationen, Leberversagen oder metabolischer Dekompensation. Einige wenige MVA-Fälle, insbesondere LateOnset-Fälle und Fälle mit nur begrenzt erhöhter Stuhlmenge, überleben unter Umständen mit zusätzlicher parenteraler Teilernährung. Eine Behandlung mit Kortikosteroiden, Kolostrum bzw. epidermalem Wachstumsfaktor war bisher nicht erfolgreich, aber bei einem Patienten erwies sich eine Octreotidbehandlung als teilweise erfolgreich [14]. Seit den 80er Jahren des 20. Jahrhunderts können die meisten betroffenen Säuglinge und Kinder, im Gegensatz zu früher, dank PE überleben. Die sich aufgrund unzulänglicher PE ergebenden Komplikationen wirken sich jedoch begrenzend auf das langfristige Überleben aus. Ferner leiden einige dieser Säuglinge und Kinder unter einem chronischen hydroelektrolytischen Ungleichgewicht und einer Azidose mit nachfolgender Wachstumsstörung, ohne dass eine assoziierte Nierenerkrankung nachgewiesen werden konnte. Andere wiederum leiden aufgrund wiederholter Episoden der Dehydra- 
Abb. 3. Intestinale Epitheldysplasie. Partielle Zottenatrophie mit Kryptenhyperplasie bzw. pseudozystischen Kryptenveränderungen, Verästelungen und Desorganisation des Oberflächenepithels.

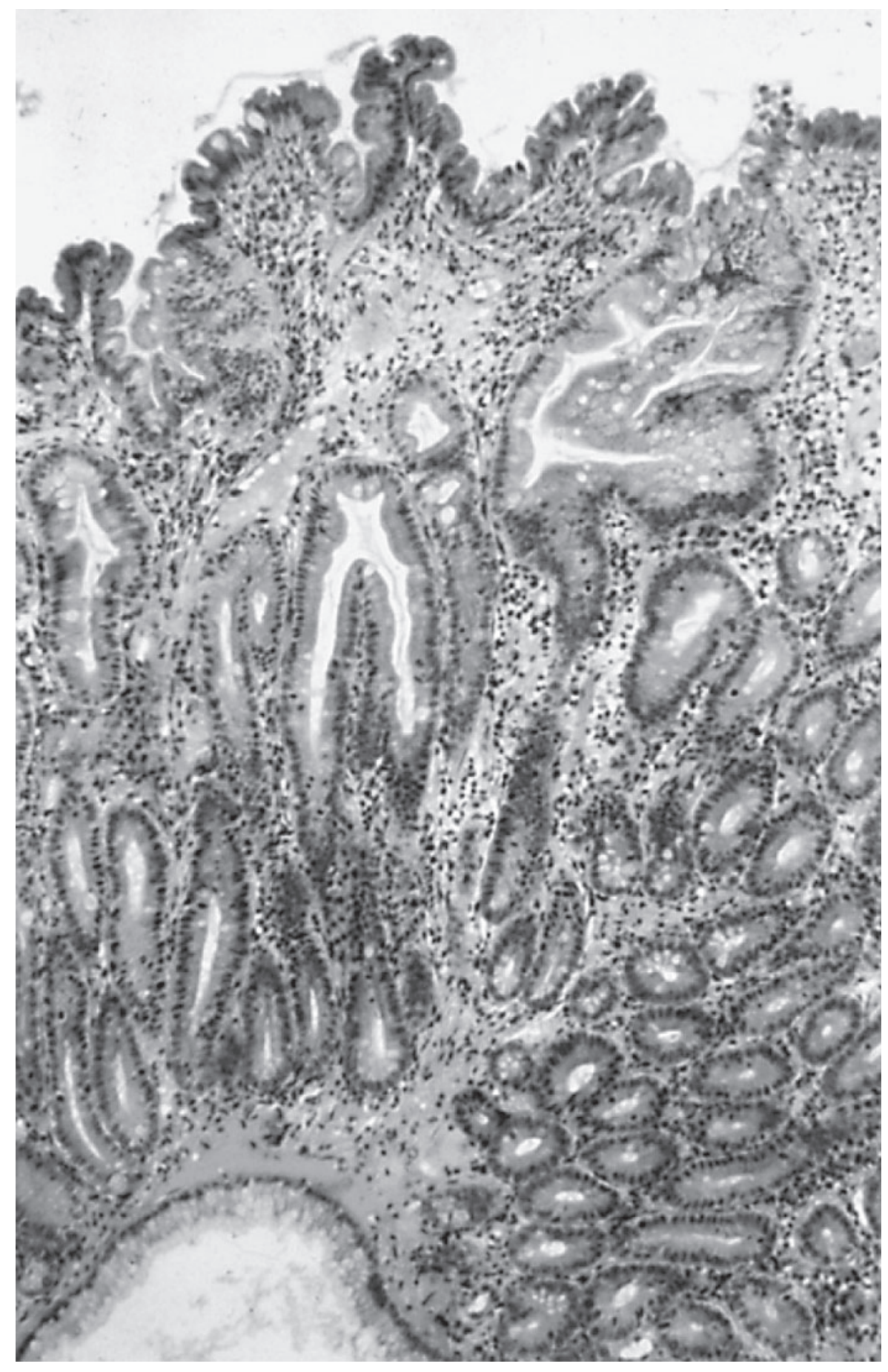

tion in Kombination mit einer unangemessenen Zufuhr von Kalziumphosphat unter Nephrokalzinose. Letztendlich persistieren hohe und unangenehme Stuhlmengen selbst bei angemessener parenteraler Dauerernährung und normalem Wachstum, erfordern in vielen Fällen einen täglichen Ersatz der verlorenen Flüssigkeit und Elek- trolyte und bergen ein hohes Risiko für schwere Dehydration. Eine Darmtransplantation stellt daher möglicherweise die einzige wirklich wirksame Behandlung dieser seltenen Darmerkrankung dar.

Es gibt Berichte über mehrere erfolgreiche Transplantationen bei MVA-Patienten [26-29]. Hierbei handelte 
es sich entweder um reine Darmtransplantationen [26] oder um kombinierte Leber-Darmtransplantationen [27, 28]. Nach wie vor umstritten ist, ob der Dickdarm zusammen mit dem Dünndarm transplantiert werden sollte. Vor kurzem berichteten wir über Patienten, die unter Early-Onset-Fällen der kongenitalen MVA litten [29]. Zwei dieser Patienten verstarben bevor sie auf die Warteliste für eine Dünndarmtransplantation gesetzt werden konnten. Ein Patient wartet noch auf die Dünndarmtransplantation. Bei 7 Patienten im Alter zwischen 3 und 11 Jahren haben wir mit Hilfe von Tacrolimus, Steroiden und IL-2-Blocker die Transplantation eines Kadaverdarms durchgeführt. Bei drei dieser Transplantationen handelte es sich um eine reine Darmtransplantation und in vier Fällen wurde eine kombinierte Leber-Darmtransplantation durchgeführt. In fünf Fällen wurde der rechte Kolon ebenfalls transplantiert (bei zwei Fällen handelte es sich um reine Dünndarmtransplantationen). Sechs der Patienten (86\%) haben überlebt, wobei die Beobachtungszeit nach der Transplantation im Mittel drei Jahre (zwischen 1 und 8 Jahren) beträgt. Keiner der Patienten musste nach der Transplantation weiterhin parenteral ernährt werden. Die Dünndarmtransplantation entweder als reine Darmtransplantation oder als kombinierte Leber-Darmtransplantation bietet MVA-Patienten erstmalig eine langfristige Perspektive [29, 30].

\section{Intestinale Epitheldysplasie oder}

Tufting-Enteropathie

Reifen et al. berichteten unter der Bezeichnung «Tufting-Enteropathie» über drei Fälle der schweren Neugeborenendiarrhoe mit Epithelveränderungen [31]. Wir stellten neun Fälle der schweren Neugeborenendiarrhoe fest, die sich eindeutig von den MVA-Fällen unterschieden [11]. Weitere Untersuchungen dieser Patienten ergaben, dass es sich bei der intestinalen Epitheldysplasie (IED) um eine konstitutive Epithelzellkrankheit handelt, die sowohl den Dünndarm als auch den Dickdarm in Mitleidenschaft zieht [32]. Nach unserer Erfahrung tritt die IED anscheinend häufig bei Patienten arabischer Herkunft, im Mittleren Osten und in Nordafrika auf. Eines der wichtigsten Merkmale dieser Erkrankung ist ihre klinische und histologische Heterogenität und ihr Zusammenhang mit Malformationen oder anderen Erkrankungen des Epithels.

\section{Klinisches Bild}

Bei den Patienten tritt typischerweise in den ersten Lebenswochen eine schwere Diarrhoe auf. Die meisten Patienten haben konsanguine Eltern bzw. Geschwister, die ebenfalls von der Krankheit betroffen sind. Einige der ebenfalls betroffenen Familienmitglieder starben in den ersten Lebensmonaten an einer schweren Diarrhoe unbekannter Ursache. In den meisten Fällen persistierte die Diarrhoe trotz Darmruhe, die Mortalitätsrate war jedoch niedriger als bei der MVA. Bei der versuchsweisen parenteralen Dauerernährung mit einem Proteinhydrolysat bzw. mit Aminosäuren verschlimmerte sich die Diarrhoe. Die Neugeborenen zeigen schnell Gedeihstörungen und entwickeln eine schwere Protein-Energie-Malnutrition.

\section{Histologisches Bild}

Bei den Patienten zeigt sich eine unterschiedlich schwere Zottenatrophie. Bei der typischen Form der Erkrankung befinden sich die Veränderungen hauptsächlich auf dem Epithel und umfassen eine Desorganisation der oberflächlichen Enterozyten mit herdartigen Ansammlungen, die wie Büschel aussehen (Abb. 3). Diese erstmals von Reifen et al. [31] beschriebenen, charakteristischen «Büschel» von herausstehendem Epithel treten bei möglicherweise bis zu 70\% der Zotten an der Zottenspitze auf. «Tufting» ist nicht auf den Dünndarm beschränkt, sondern tritt auch in der Schleimhaut des Dickdarms auf [11]. Dieses Erscheinungsbild lässt sich auch im Kryptenepithel beobachten und die Krypten erscheinen ferner häufig verändert mit Dilatation, wie z.B. Pseudozysten, und abnormer Regeneration mit Verästelungen [11]. Eine Untersuchung der Komponenten der Grundmembran ergab eine im Vergleich zu Biopsieproben von Zöliakiepatienten oder Patienten mit Autoimmun-Enteropathie abnorme Ablagerung von Laminin und Heparansulfat-Proteoglykan auf dieser Ebene [11]. Im Vergleich zu Kontrollpersonen zeigte sich an der Schnittstelle zwischen Epithel und Lamina propria eine schwache und unregelmässige Lamininablagerung bei gleichzeitig grosser, lamellenartiger Ablagerung von HeparansulfatProteoglykan, die auf eine abnorme Entwicklung der Grundmembran hindeutet. Diese Ablagerungen sind möglicherweise die Ursache der Epithelveränderungen. Andererseits beobachteten wir bei der IED eine erhöhte immunhistochemische Expression von Desmoglein sowie ultrastrukturelle Veränderungen in den Desmosomen, die verlängert waren und in einer höheren Zahl vorlagen [32] (Abb. 4).

Wird eine MVA aufgrund mikroskopischer Untersuchungen von Biopsieproben mit PAS-Färbung ausgeschlossen, so fällt der Verdacht am häufigsten auf die IED. Eine endgültige Diagnose dieser Erkrankung erfolgt nach wiederholten Darmbiopsien, die sich von subnor- 


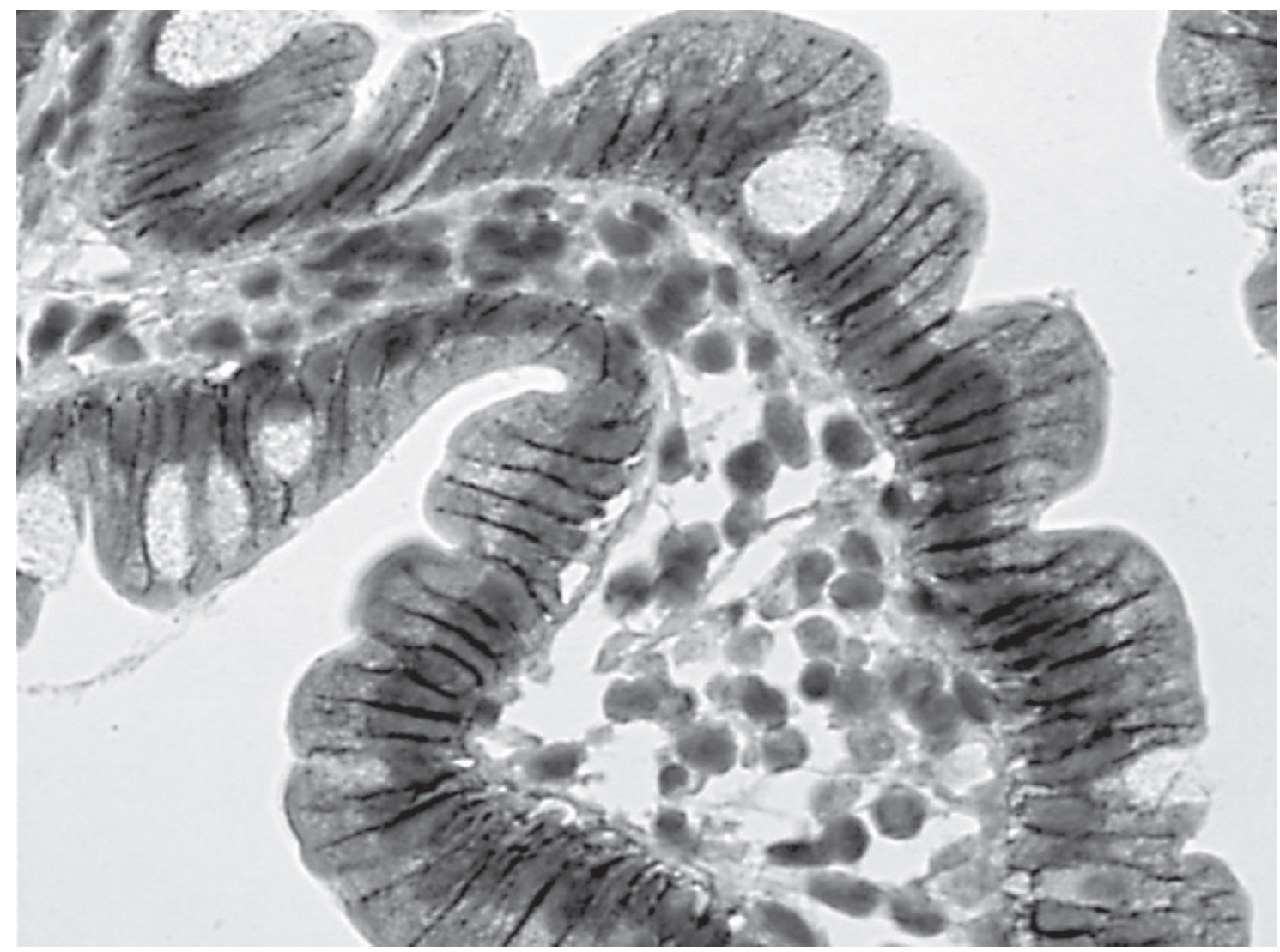

Abb. 4. Intestinale Epitheldysplasie. Färbung der erhöhten Expression von Desmoglein an der undurchlässigen Verbindung (Tight Junction) bei einem Patienten mit intestinaler Epitheldysplasie.

mal im frühen Lebensstadium (lediglich unspezifische Zottenatrophie mit oder ohne monozelluläre Infiltration der Lamina propria) zu den charakteristischen Bündeln verändern. Eine weitere Schwierigkeit entsteht durch die Infiltration der Lamina propria durch die T-Zellen. TZell-Infiltrate bekräftigen, wie bereits beschrieben, die Diagnose einer mit dem Immunsystem in Zusammenhang stehenden Enteropathie, insbesondere wenn keine Büschel festgestellt werden. Die Darmdurchlässigkeit wird möglicherweise durch eine Störung der Zelladhäsion erhöht und führt in der Folge zu einer entzündlichen Reaktion. Murch et al. [33] haben diese Art von Läsion bei Säuglingen mit Epitheldysplasie beschrieben.

\section{Erkrankungen, die mit der IED in Zusammenhang} stehen

Es wurden mehrere Fälle berichtet, bei denen die IED mit bestimmten phänotypischen Anomalien, z.B. dem Dubowitz-Syndrom oder dem Missbildungssyndrom, assoziiert war [34, 35]. Vor kurzem wurde bei vier Kindern ein Zusammenhang zwischen der kongenitalen intraktablen Diarrhoe im Säuglingsalter und der Choanalatresie gemeldet [34]. Wir haben Missbildungen, u.a. Rektum- oder Ösophagusatresie, beobachtet und vor kurzem berichtet, dass bei ungefähr 60\% der Patienten eine unspezifische punktförmige Keratitits festzustellen ist [36]. Diese Tatsache ist äusserst interessant, da es sich bei der Keratitis ebenfalls um eine Epithelerkrankung handelt, die unter Umständen dazu beitragen kann, die der intestinalen Epithelerkrankung zugrunde liegenden molekularen Mechanismen aufzuklären. Die Tatsache, dass bei einigen Kindern keine ophthalmologischen Symptome festzustellen sind, veranschaulicht die wahrscheinliche Heterogenität der Erkrankung.

Lachaux et al. [37] haben interessanterweise vor kurzem über den Fall eines Neugeborenen mit Pylorusatresie und intraktabler Diarrhoe berichtet. In der lichtmikroskopischen Untersuchung zeigten sich ausgedehnte Abschuppungen vom Fundus bis zum Rektum mit nur einigen wenigen verbleibenden Epithelzellen am Krypten- 
boden. Die Untersuchung des Darmepithels im Elektronenmikroskop zeigte zwar normale Desmosomen, jedoch einen Spalt zwischen der Lamina propria und dem basalen Pol der Enterozyten. Bei diesem ersten beschriebenen Fall bestand ein Zusammenhang mit einem angeborenen Mangel an $\alpha 6 \beta 4$-Integrin, das bekanntermassen auch bei der Epidermolysis bullosa in Form eines Defekts des Gens für $\alpha 6 \beta 4$-Integrin eine Rolle spielt.

\section{Pathophysiologie und Übertragungsart}

Es wurde der Nachweis erbracht, dass die IED, im Vergleich zu Biopsieproben von Patienten mit Zöliakie oder Autoimmun-Enteropathie, mit einer veränderten Basalmembran zusammenhängt [11]. Die Moleküle der Grundmembran sind an den epithelial-mesenchymalen Zelleninteraktionen beteiligt, die für die Entwicklung und Differenzierung des Darms von grösster Wichtigkeit sind [38]. Bei IED-Patienten wurden Veränderungen beobachtet, die auf veränderte Zell-Zell- und Zell-MatrixInteraktionen hindeuten, ohne dass Anomalien in der Polarisierung bzw. Proliferation der Epithelzellen nachgewiesen werden konnten [32]. Zu diesen Veränderungen zählen eine abnorme Verteilung der Adhäsionsmoleküle und des $\alpha 2 \beta 1$-Integrins entlang der Krypten-Zotten-Achse. Das $\alpha 2 \beta 1$-Integrin ist an der Kommunikation der Epithelzellen mit diversen Komponenten der Grundmembran, wie z.B. Laminin und Kollagen, beteiligt. Welche pathophysiologischen Mechanismen zu einer erhöhten immunhistochemischen Expression von Desmoglein führen und welche ultrastrukturellen Veränderungen sich in den Desmosomen ergeben ist nach wie vor unklar [32]. Eine Maus mit fehlender Funktionsänderung in dem für den Transkriptionsfaktor Elf3 kodierenden Gen weist morphologische Merkmale auf, die den bei Säuglingen mit Epitheldysplasie beobachteten Merkmalen ähneln [39].

Bei diesem Mäusemodell liegt eine abnorme Morphogenese der Zotten vor, wohingegen die progenitorischen Kryptenzellen normal erscheinen. Bei Mäusen mit einem Elf3-Mangel produzieren die Enterozyten niedrige Konzentrationen des Typ-2-Rezeptors für den transformierenden Wachstumsfaktor $\beta$, der die Differenzierung des unreifen intestinalen Epithels induziert. Beim menschlichen Säugling wurde der primäre oder sekundäre Charakter der beobachteten Veränderungen bislang noch nicht bestimmt. Das Vorliegen von Büscheln bietet unter Umständen wichtige Hinweise. Bei den Büscheln handelt es sich um nicht-apoptotische, nicht länger mit der Grundmembran in Kontakt stehende Epithelzellen an den Zottenspitzen. Zwar handelt es sich hier um eine rein speku- lative Vermutung, aber man nimmt an, dass dieser Effekt möglicherweise auf eine gestörte Apoptose der Enterozyten am Ende ihrer Lebensspanne bzw. eine Veränderung der interzellulären Kommunikation zurückzuführen ist. Der primäre oder sekundäre Charakter der Büschelbildung ist noch zu bestimmen.

Aufgrund des eindeutigen Zusammenhangs mit einer Konsanguinität der Eltern bzw. weiteren betroffenen Geschwistern vermutet man bis jetzt, dass diese Krankheit genetischen Ursprungs ist. Diese Merkmale lassen auf einen autosomal rezessiven Erbgang schliessen. Ein an dieser kongenitalen, autosomal rezessiv vererbten Erkrankung beteiligtes Gen wurde bisher nicht identifiziert. Diese Enteropathie tritt insbesondere bei Populationen aus dem Mittleren Osten häufiger auf als die MVA.

\section{Outcome}

Die therapieresistente Neugeborenendiarrhoe erfordert eine parenterale Langzeiternährung. Anscheinend jedoch entwickeln manche Säuglinge einen milderen Phänotyp als andere [40]. Dank teilweiser Restdarmfunktion und nur begrenzt erhöhter Stuhlmenge benötigen manche Patienten langfristig nur eine parenterale Teilernährung in Form von drei- bis viermal wöchentlich verabreichten Infusionen. Diese Patienten sollten jedoch sorgfältig überwacht werden, um eine progressive Wachstumsverzögerung zu vermeiden. Die meisten Patienten sind jedoch aufgrund des Schweregrads ihrer intestinalen Malabsorption und Diarrhoe ausschliesslich auf eine tägliche parenterale Langzeiternährung angewiesen und dem damit zusammenhängenden Komplikationsrisiko ausgesetzt. Die IED kann eine weitere Indikation für eine Darmtransplantation sein [41, 42].

\section{Weitere kongenitale Enteropathien}

Die sogenannte «phänotypische Diarrhoe» gehört zum Syndromkomplex der intraktablen Diarrhoe im Säuglingsalter, hängt mit phänotypischen Anomalien und einem Immunmangel zusammen und ist ebenfalls eine seltene Erkrankung, über die erst seit kurzem berichtet wird [12]. Bei den betroffenen Patienten treten innerhalb der ersten sechs Lebensmonate (in den meisten Fällen im Alter von unter einem Monat) Diarrhoe sowie diverse weitere, identische klinische Merkmale auf. Es handelt sich um SGA-Kinder mit einem abnormen Phänotyp [12]. Bei allen diesen Kindern liegt ein fazialer Dysmorphismus mit vorstehender Stirn, breiter Nase und Hypertolerismus vor. Die Kinder haben eine ausgeprägte Haarveränderung, Trichorrhexis nodosa, mit weichem, schwer $\mathrm{zu}$ bändigendem und schlecht pigmentiertem 
Haar, das leicht ausgeht. Diese Merkmale werden auch bei Kindern aus dem Mittleren Osten beobachtet. Die Trichorrhexis nodosa ist eine sehr häufige Form der kongenitalen Haardysplasie und kann bei mehreren Erkrankungen auftreten [43, 44]. Die Patienten weisen ferner trotz normaler Immunglobulinkonzentrationen im Blutserum eine unzureichende Reaktion auf Antikörper auf und auch die Hauttests auf bestimmte Antigene zeigen trotz positiv proliferativer Reaktionen in vitro unzureichende Ergebnisse [12]. Untersucht man die aus dem Dünndarm von Patienten mit syndromatischer Diarrhoe entnommenen Biopsieproben, so zeigt sich eine moderate bis schwere Zottenatrophie mit unbeständigen mononukleären Zell-infiltraten der Lamina propria ohne Epithelveränderungen. Histologisch sind keine spezifischen Anomalien festzustellen. Die Prognose gestaltet sich bei dieser Art von intraktabler Diarrhoe des Säuglingsalters schlecht, da die meisten Patienten im Alter zwischen zwei und fünf Jahren sterben. Bei einigen dieser Patienten treten Early-Onset-Erkrankungen der Leber auf [12]. Die Ursache dieser Diarrhoe ist unbekannt und die Beziehungen zwischen niedrigem Geburtsgewicht, Dysmorphismus, schwerer Diarrhoe, Trichorrhexis und Immunmangel sind unklar.

\section{Management des Darmversagens}

\section{Parenterale Langzeiternährung}

Ob im Krankenhaus oder zu Hause, die parenterale Langzeiternährung setzt den Patienten mehreren Komplikationen aus, u.a. der Kathetersepsis und der Thrombose, Leber- und Knochenkrankheiten [45]. Die tägliche Katheterpflege und die Prävention septischer Komplikationen sind von grösster Wichtigkeit [46]. Aufgrund des Risikos der Cholestase und Leberschädigung sollten regelmässig Leberfunktionstests durchgeführt werden. Bei Patienten, bei denen die Gallenblase nicht entfernt wurde, sollten mindestens zweimal jährlich Ultraschalluntersuchungen durchgeführt werden, um nach eingedickter Gallenflüssigkeit (Sludge) und Gallensteinen zu suchen. Ferner sollte die Knochenmineralisierung mit Hilfe von Röntgenabsorptions-Densitometrie (DEXA, Dual Energy X-ray Absorptiometry) untersucht, der Phosphat- und Kalziumstatus bewertet und für ein sorgfältiges Ernährungsmanagement gesorgt werden, um eine mit der PE-verbundene Knochenerkrankung zu vermeiden.
Faktoren und Management von Lebererkrankungen

Lebererkrankungen sind die bei Patienten mit Darmversagen am häufigsten auftretenden und schwersten Komplikationen. Die Bezeichnung «PE-assoziierte Lebererkrankung» müsste eigentlich durch die Bezeichnung «mit Darmversagen assoziierte Lebererkrankung» abgelöst werden. Tatsächlich sind die wichtigsten Faktoren, die zu Cholestase und Lebererkrankung im Endstadium führen, mit verminderter Darmfunktion und bakteriellem Überwuchs verbunden. Eine unzureichende PE verschlimmert den Leberschaden.

$\mathrm{Zu}$ den Hauptfaktoren, die mit einer Lebererkrankung assoziiert werden, zählen:

(1) Die Unterbrechung des enterohepatischen Kreislaufes (Erkrankung oder Resektion des Ileums) [47, 48].

(2) Enterostase mit nachfolgend bakteriellem Überwuchs bzw. bakterieller Translokation intraluminal (Endotoxinämie) [49].

(3) Rezidivierende Episoden der Kathetersepsis [50, 51].

(4) Frühgeburt [51].

(5) Unangemessene Aufnahme von Makronährstoffen, z.B. bei PE in Form einer Dauerinfusion mit übermässiger Glukoseaufnahme, die zu Hyperinsulinismus mit nachfolgender Steatose [52] und unzureichender Versorgung mit Aminosäuren führt [49].

(6) Übermässige Aufnahme langkettiger, mehrfach ungesättigter Fettsäuren mit nachfolgender Lipidperoxidation [53, 54] und Aufnahme eines hohen Gehalts an Phytosterolen aus Emulsionen auf Sojaölbasis [55].

(7) Eine zu hohe Aluminium-, Eisen- bzw. Chromaufnahme [56-58].

Bei Frühgeburten bzw. SGA-Kindern mit schwerer nekrotisierender Enterocolitis ist das Risiko, aufgrund der Kombination von Frühgeburt, teilweisem Darmverschluss, gramnegativer Sepsis, protrahierter Darmruhe, kontinuierlich anstatt zyklisch infundierter PE und wiederholter Kathetersepsis, eine Lebererkrankung bzw. ein frühes Leberversagen im Endstadium zu entwickeln besonders hoch.

Durch folgende Massnahmen lässt sich ein Leberschaden möglicherweise verhindern oder beheben:

(1) Stimulation des Dünndarms und der Gallenwege, indem man die Aufnahme von langkettigen Triglyceriden oder Muttermilch sicherstellt oder CCK (Cholecystokinin)-Analoga injiziert [59].

(2) Suppression des durch die Enterostase verursachten bakteriellen Überwuchs durch Verabreichung von Metronidazol [60] bzw. Durchführung einer Enteroplastik zur Verengung der Dilatation. 
(3) Einnahme von Ursodesoxycholsäure $(30 \mathrm{mg} / \mathrm{kg} /$ Tag) zur Reduktion des Leberschadens [61].

(4) Verhinderung einer Kathetersepsis durch entsprechende tägliche Pflege des Schlauchs [50, 51].

(5) Richtige Einstellung der Nährstoffaufnahme mittels PE durch:

(a) Einschränkung der Glukoseaufnahme zur Verringerung der Fettansammlungen in der Leber [62, 63], und

(b) Verwendung einer geeigneten intravenösen Fettemulsion in der geeigneten Menge, um essentielle Fettsäuren bereitzustellen, die Glucoseaufnahme zu senken und die Peroxidation zu begrenzen [53-55]. Mit den vor kurzem entwickelten Fettemulsionen auf Olivenölbasis lässt sich die Peroxidation unter Umständen verringern [54]. Die täglich intravenös verabreichte Lipidmenge sollte maximal $2-2,5 \mathrm{~g} / \mathrm{kg}$ betragen.

(6) Steuerung der bereitgestellten Lipidmenge und der Geschwindigkeit der Lipidzufuhr bzw. Unterbrechung der intravenösen Lipidzufuhr, sobald sich eine Thrombozytopenie, Hyperbilirubinämie bzw. Gelbsucht abzeichnet [53].

(7) Verwendung neuer, für den Einsatz in der Pädiatrie adaptierter Aminosäurelösungen, die geeignete Aminosäuren sowie Taurin bereitstellen [49].

(8) Zyklische Verabreichung der PE zur Verringerung von Hyperinsulinismus und Lebersteatose [64].

(9) Anpassung der Eisenaufnahme und Senkung des Aluminiumgehalts der PE Lösungen [65].

Bei entsprechender Sorgfalt und Prävention lassen sich Lebererkrankungen verzögern oder sogar vermeiden und der Ernährungs- bzw. Allgemeinzustand des Patienten unter Umständen soweit erhalten, dass bei einer aufgrund eines irreversiblen Darmversagens eventuell erforderlichen reinen Dünndarmtransplantation die Prognosen besser sind.

\section{Darmtransplantation}

Was auch immer die Ursache eines protrahierten oder irreversiblen Darmversagens ist, die parenterale Langzeiternährung ist die Hauptstütze der Therapie. Die PE zu Hause bietet den Säuglingen und Kindern die Möglichkeit, in der für sie psychisch besten Umgebung aufzuwachsen $[66,67]$. Bevor eine Darmtransplantation in Betracht gezogen werden kann, muss die Irreversibilität des Darmversagens belegt worden sein.

Sobald die Darmtransplantation als therapeutische Option indiziert ist, müssen Transplantationschirurgen und pädiatrische Gastroenterologen umfassende multi- disziplinäre Diskussionen führen. Die Bewertungen und Entscheidungen basieren auf dem möglichen Eintreten der beim International Symposium on Small Bowel Transplantation in Miami im September 2003 aufgezählten Komplikationen. Diese entsprechen den im Positionspapier der American Society of Transplantation [68] enthaltenen Kriterien und sind nach wie vor umstritten; dies gilt insbesondere für die Kriterien, die sich auf vaskuläre Thrombose und Sepsis beziehen:

(1) Lebererkrankung (Bilirubinkonzentrationen $>3 \mathrm{mg} / \mathrm{dl}$, Splenomegalie, Thrombozytopenie, Ösophagusvarizen, Koagulopathie, Stomablutung, hepatische Fibrose oder Zirrhose).

(2) Thrombose der Hauptvenenzugänge ( $>2$ Thrombosen in den Schlüsselbein- (V. subclavia), Hals- (V. jugularis) oder Oberschenkelvenen (V. femoralis)). Wir legen in der Regel einen Zugang links im oberen Hohlvenenbett.

(3) Rezidivierende Episoden der Kathetersepsis (2 Episoden von systemischer Sepsis sekundär nach Infektion in Verbindung mit dem Katheter pro Jahr, 1 Episode der mit dem Katheter verbundener Fungämie, septischem Schock bzw. akutem Atemnots-Syndrom (ARDS)).

(4) Unkontrollierte Flüssigkeits- und Elektrolytverluste sowie häufige, schwere akute Dehydration aufgrund der zugrunde liegenden ursächlichen Erkrankung.

Diese Kriterien stellen Richtlinien, keine offiziellen Empfehlungen dar. Sie müssen mit dem Risiko einer Darmtransplantation verglichen und genau abgewogen werden.

Für eine Darmtransplantation gelten die gleichen Kontraindikationen wie für andere Organe. Aufgrund der mit einer Darmtransplantation verbundenen Risiken, insbesondere bei schlechtem Allgemeinzustand des Transplantatempfängers zum Zeitpunkt der Transplantation und kleinem Spenderpool, ist es nach wie vor von grösster Wichtigkeit, dass sich dem Patienten durch die Transplantation potenziell eine eindeutige Nutzwirkung erschliesst. $\mathrm{Zu}$ den weiteren Kontraindikationen zählen kongenitale oder erworbene neurologische Behinderungen, lebensbedrohliche Erkrankungen ausserhalb des Verdauungstrakts, nicht resektable maligne Tumoren sowie eine vaskuläre Durchgängigkeit, die nicht ausreicht, um für eine Dauer von bis zu 6 Monaten nach der Transplantation einen einfachen zentralen Venenzugang zu gewährleisten.

\section{Arten der Transplantation}

Patienten mit irreversiblem Darmversagen und Lebererkrankung im Endstadium (Leberzirrhose) sind eindeutig Kandidaten für lebensrettende Verfahren, wie z.B. 
eine kombinierte Leber-Darmtransplantation (LITx). Bei Patienten mit schwerer hepatischer Fibrose gestaltet sich das Management schwieriger. Hier sind innerhalb von 6-12 Monaten wiederholte Leberbiopsien sowie sorgfältige Beurteilungen des Pfortaderhochdrucks (portale Hypertonie) nötig. Ferner gestaltet sich die richtige Einschätzung, wie viel funktionelles Lebergewebe nötig ist, um die im Rahmen der Transplantation durchgeführte Arterialisierung der Pfortader zu überstehen, schwierig. Kinder mit schwerer fortgeschrittener und progressiver hepatischer Fibrose sind normalerweise Kandidaten für eine LITx. Bei einigen PE-abhängigen Patienten mit fortgeschrittener Leberdysfunktion tritt unter Umständen parallel mit einer autologen Darmerhaltung eine Erholung der Leberfunktion und der biochemischen Leberwerte ein. Bei mehreren konsekutiven Fällen der schweren Cholestase trat nach Absetzen der intravenös verabreichten Lipide eine vollständige Erholung ein [69]. Die Histologie ist nicht immer prädiktiv für eine Erholung der Leberfunktion [70]. Wird erst einmal eine Zirrhose festgestellt, so beträgt die Ein-Jahres-Überlebensrate jedoch nur 20-30\% [71].

\section{Zeitpunkt für die Indikationsstellung zur \\ Darmtransplantation}

Die Faktoren, die sich auf das Überleben von Kindern mit Darmversagen auswirken, bei denen die Indikation zur Darmtransplantation gestellt wurde, sind allgemein bekannt und umfassen: Alter $<1 \mathrm{Jahr}$; chirurgisch behandelbare Erkrankung, Brückenfibrose bzw. Zirrhose; Bilirubinspiegel von $>3 \mathrm{mg} / \mathrm{dl}$ und Thrombozytopenie [71]. Es steht unzweifelhaft fest, dass Patienten, die zur kombinierten Leber-Darmtransplantation an ein intensivmedizinisches Zentrum verwiesen werden, geschwächt sind, unter multiplen Komplikationen leiden und längere Krankenhausaufenthalte benötigen. Der UNOS-Bericht weist darauf hin, dass die Mortalität bei Patienten, die auf den Wartelisten für eine Darmtransplantation stehen, höher ist als bei Patienten, die auf andere Transplantationen warten [72]. Dies mag als Erklärung für die in diversen Studien gemeldeten, schlechten Überlebensraten der Patienten und der Transplantate dienen [73]. Das Intestinal Transplant Registry bestätigte, dass Patienten, die zu Hause auf die Transplantation warten, bei Transplantationen eine bessere Ein-Jahres-Überlebensrate aufwiesen als Patienten, die im Krankenhaus auf die Transplantation warten (74 gegenüber 59\%; $<<0,00001)$ [73] Die Tendenz, Transplantationen proportional gesehen bei mehr Patienten durchzuführen, die zu Hause auf die Transplantation warten, ist einer der Hauptfaktoren, der zu den in der letzten Zeit verbesserten Transplantat- und Patientenüberlebensraten beitrug [73].

Nur wenige medizinische Zentren bieten für alle Stadien des Darmversagens, vom Einsetzen bis zur Darmtransplantation, geeignete Behandlungsprogramme, einschliesslich Programme für die PE zu Hause. Der klinischeZustand von Kindern, die aus medizinischen Zentren mit und aus medizinischen Zentren ohne Expertenteams für die PE zur Darmtransplantation überwiesen wurden, wies grosse Unterschiede auf [74]. Die langfristige Behandlung des Darmversagens hat sich, wie man anhand der in letzter Zeit veröffentlichten Studien sehen kann, $\mathrm{zu}$ einem sehr wichtigen Problem entwickelt [75]. Ein nationales oder internationales Netzwerk könnte Informationen über das Darmversagen liefern, auf die sich die Anforderungen der Gesundheitspflege stützen [76] und die eine Optimierung des Managements, eine Verbesserung des medizinischen Ansatzes und die Förderung der Grundlagen- und der angewandten Forschung in diesem Bereich ermöglichen sollten.

Ein geeignetes Management des Darmversagens erfordert in der Tat Referenzzentren mit auf einem multidisziplinären Ansatz beruhenden Strategien unter Beteiligung von pädiatrischen Gastroenterologen, Ernährungswissenschaftlern, pädiatrischen Chirurgen, spezialisierten Krankenschwestern, Diätetikern, Sozialarbeitern und Psychologen. Dieser ganzheitliche Ansatz sollte auf jede Art und auf jedes Stadium des Darmversagens angepasst sein, und auch die PE zu Hause sowie ein Transplantationsprogramm umfassen.

\section{Schlussfolgerung}

Ein frühes, in Referenzzentren durch eine multidisziplinäre Expertengruppe sichergestelltes Management des Darmversagens ist der Schlüssel zur frühzeitigen Erkennung des Darmversagens, seiner Reversion, verbesserten Outcomes und früheren Darmtransplantationen. Wie eine Arbeitsgruppe zur pädiatrischen Gastroenterologie und Ernährung vor kurzem in ihrem Bericht betonte, bedarf es in allen Bereichen der Adaption nach Dünndarmresektion noch weiterer Forschungsarbeiten und der Identifizierung von Genen, die an der kongenitalen Enteropathie bzw. dem intestinalen Pseudo-Obstruktionssyndrom beteiligt sind [77]. Die für die langfristige PE zu Hause und die Darmtransplantation jeweils entstehenden Kosten sind ebenfalls zu bewerten, um die beste Option zur Maximierung der Lebensqualität der betroffenen Kinder und ihrer Familien anzustreben. 


\section{Literatur}

1 Nordgarrd I, Hansen BS, Mortensen PB: Importance of colonic support for energy absorption as small-bowel failure proceeds. Am J Clin Nutr 1996;64:222-231.

2 Crenn P, Coudray-Lucas C, Thuillier F, Cynober L, Messing B: Postabsorptive plasma citrulline concentration is a marker of absorptive enterocyte mass and intestinal failure in humans. Gastroenterology 2000;119:14961505.

-3 Crenn P, Vahedi K, Lavergne-Slove A, Cynober L, Matuchansky C, Messing B: Plasma citrulline: A marker of enterocyte mass in villous atrophy-associated small bowel disease. Gastroenterology 2003;124:1210-1219.

-4 Pappas PA, Saudubray JM, Tzakis AG, et al: Serum citrulline and rejection in small bowel transplantation: a preliminary report. Transplantation 2001;72:1212-1216.

5 Dabbas-Tyan M, Colomb V, Rosilio M, Landais P, Ricour C, Goulet O: Evaluation of the effect of recombinant human growth hormone (rhGH) treatment of children with short bowel syndrome. J Pediatr Gastroenterol Nutr 2000; 31:S165-S166.

6 Walker-Smith JA: Diarrhoeal disease: current concepts and future challenges. Malnutrition and infection. Trans R Soc Trop Med Hyg 1993;87(suppl 3):13-15.

-7 Goulet O, Révillon Y, Jan D, et al: Neonatal short bowel syndrome. J Pediatr 1991;119:18 23.

-8 Goulet O, Ruemmele F, Lacaille F, Colomb V: Irreversible intestinal failure. J Pediatr Gastroenterol Nutr 2004;38:250-269.

$\checkmark 9$ Cuenod B, Brousse N, Goulet O, et al: Classification of intractable diarrhea in infancy using clinical and immunohistological criteria. Gastroenterology 1990;99:1037-1043.

10 Goulet O, Brousse N, Canioni D, et al: Syndrome of intractable diarrhoea with persistent villous atrophy in early childhood: A clinicopathological survey of 47 cases. J Pediatr Gastroenterol Nutr 1998;26:151-161.

-11 Goulet O, Kedinger M, Brousse N, et al: Intractable diarrhea of infancy: a new entity with epithelial and basement membrane abnormalities. J Pediatr 1995;127:212-219.

12 Giraut D, Goulet O, Ledeist F, et al: Intractable diarrhea syndrome associated with phenotypic abnormalities and immune deficiency. J Pediatr 1994;125:36-42.

13 Davidson GP, Cutz E, Hamilton JR, Gall DG: Familial enteropathy: a syndrome of protracted diarrhea from birth, failure to thrive, and hypoplastic villous atrophy. Gastroenterology 1978;75:783-790.

14 Phillips AD, Schmitz J: Familial microvillous atrophy: A clinicopathological survey of 23 cases. J Pediatr Gastroenterol Nutr 1992;14: 380-396.

15 Holmberg C: Congenital chloride diarrhea. Clin Gastroenterol 1980;3:583-602.
16 Booth IW, Stange G, Murer H, Fenton TR, Milla PJ: Defective jejunal brush-border $\mathrm{Na}+$ / $\mathrm{H}+$ exchange: a cause of congenital secretory diarrhoea. Lancet 1985;i:1066-1069.

17 Phillips AD, Jenkins P, Raafat F, WalkerSmith JA: Congenital microvillous atrophy: specific diagnostic features. Arch Dis Child 1985;60:135-140.

18 Phillips AD, Szfranski M, Man L-Y, Wall W: Periodic acid Schiff staining abnormality in microvillous atrophy: photometric and ultrastructural studies. J Pediatr Gastroenterol Nutr 2000;30:34-42.

19 Phillips A, Fransen J, Hauri HP, Sterchi E: The constitutive exocytotic pathway in microvillous atrophy. J Pediatr Gastroenterol Nutr 1993;17:239-246.

20 Groisman GM, Amar M, Livne E: CD10: A valuable tool for the light microscopic diagnosis of microvillous inclusion disease (familial microvillous atrophy). Am J Surg Pathol 2002; 26:902-907.

21 Youssef N, Canioni D, Ruemmele F, et al: CD10 expression in microvillous inclusion disease (abstract). J Pediatr Gastroenterol Nutr 2003; 36:563.

22 Raafat F, Green NJ, Nathavitharana KA, Booth IW: Intestinal microvillous dystrophy: a variant of microvillous inclusion disease or a new entity? Hum Pathol 1994;25:1243-1248.

-23 Phillips AD, Brown A, Hicks S, Schuller S, Murch SH, Walker-Smith JA, Swallow DM: Acetylated sialic acid residues and blood group antigens localise within the epithelium in microvillous atrophy indicating internal accumulation of the glycocalyx. Gut 2004;53:17641771.

24 Nathavitharana KA, Green NJ, Raafat F, Booth IW: Siblings with microvillous inclusion disease. Arch Dis Child 1994;71:71-73.

25 Heinz-Erian P, Schmidt H, Le Merrer M, et al: Congenital microvillous atrophy in a girl with autosomal dominant hypochondroplasia. J Pediatr Gastroenterol Nutr 1999;28:203-205.

26 Oliva MM, Perman JA, Saavedra JM, et al: Successful intestinal transplantation for microvillous inclusion disease. Gastroenterology 1994; 106:771-774.

27 Herzog D, Atkinson P, Grant D, et al: Combined bowel-liver transplantation in an infant with microvillous inclusion disease. J Pediatr Gastroenterol Nutr 1996;22:405-408.

28 Randak C, Langnas AN, Kaufman SS, et al: Pretransplant management and small bowelliver transplantation in an infant with microvillous inclusion disease. J Pediatr Gastroenterol Nutr 1998;27:333-337.

29 Ruemmele FM, Jan D, Lacaille F, et al: New perspectives for children with microvillous inclusion disease: early small bowel transplantation. Transplantation 2004;77:1024-1028.

30 Croft NM, Howatson AG, Ling SC, et al: Microvillous inclusion disease: an evolving condition. J Pediatr Gastroenterol Nutr 2000;31: 185-189.
31 Reifen RM, Cutz E, Griffiths AM, Ngan BY, Sherman PM: Tufting enteropathy: a newly recognized clinicopathological entity associated with refractory diarrhea in infants. J Pediatr Gastroenterol Nutr 1994;18:379-385.

32 Patey N, Scoazec JY, Cuenod-Jabri B, et al: Distribution of cell adhesion molecules in infants with intestinal epithelial dysplasia (tufting enteropathy). Gastroenterology 1997;113: 833-843.

33 Murch S, Graham A, Vermault A, et al: Functionally significant secondary inflammation occurs in a primary epithelial enteropathy. $\mathrm{J}$ Pediatr Gastroenterol Nutr 1997;24:467.

- 34 Krantz M, Jansson U, Rectors S, Ryd W, Kristiansson B: Hereditary intractable diarrhea with choanal atresia. A new familial syndrome. J Pediatr Gastroenterol Nutr 1997;24:470.

35 Abely M, Fromont Hankard G, et al: Intractable infant diarrhea with epithelial dysplasia associated with polymalformation. J Pediatr Gastroenterol Nutr 1998;27:348-352.

36 Djeddi D, Verkarre V, Talbotec C, et al: Tufting enteropathy and associated disorders (abstract). J Pediatr Gastroenterol Nutr 2002;34: 446.

37 Lachaux A, Bouvier R, Loras I, et al: Isolated deficient $\alpha 6 \beta 4$ integrin expression in the gut associated with intractable diarrhoea. J Pediatr Gastroenterol Nutr 1999;29:395-401.

- 38 Simon-Assmann P, Kedinger M: Heterotypic cellular cooperation in gut morphogenesis and differentiation. Cell Biol 1993;4:221-230.

- 39 Ayn NG, Waring P, Ristevski S, et al: Inactivation of the transcription factor Elf3 in mice results in dysmorphogenesis and altered differentiation of intestinal epithelium. Gastroenterology 2002;122:1455-1466.

40 Cameron DJS, Barnes GL: Successful pregnancy outcome in tufting enteropathy. J Pediatr Gastroenterol Nutr 2003;36:158

41 Lacaille F, Cuenod B, Colomb V, et al: Successful combined liver and small bowel transplantation in a child with epithelial dysplasia. J Pediatr Gastroenterol Nutr 1998;27:230-233.

42 Paramesh AS, Fishbein T, Tschernia A, et al: Isolated small bowel transplantation for tufting enteropathy. J Pediatr Gastroenterol Nutr 2003;36:138-140.

43 Itin PH, Pittelkow MR: Trichothiodystrophy: review of sulfur-deficient brittle hair syndromes and association with the ectodermal dysplasia. J Am Acad Dermatol 1990;22:705717.

44 Verloes A, Lombet J, Lambert Y, et al: Trichohepato-enteric syndrome: further delineation of a distinct syndrome with neonatal hemochromatosis phenotype, intractable diarrhea, and hair anomalies. Am J Med Genet 1997;68: 391-395.

45 Shulman RJ, Phillips S: Parenteral nutrition in infants and children. J Pediatr Gastroenterol Nutr 2003;36:587-607. 
-46 Colomb V, Fabeiro M, Dabbas M, Goulet O, Merckx J, Ricour C: Central venous catheterrelated infections in children on long-term home parenteral nutrition: incidence and risk factors. Clin Nutr 2000;19:355-359.

-47 Colomb V, Goulet O, Rambaud C, et al: Long term parenteral nutrition in children: liver and gallbladder disease. Transplant Proc 1992;24: 1054-1055.

-48 Moss RL, Das JB, Raffensperger JG: Total parenteral nutrition-associated cholestasis: clinical and histopathological correlation. J Pediatr Surg 1993;28:1270-1275.

49 Forchielli ML, Walker WA: Nutritional factors contributing to the development of cholestasis during total parenteral nutrition. Dev Pediatr 2003;50:245-267

-50 Colomb V, Jobert-Giraud A, Lacaille F, Goulet O, Fournet JC, Ricour C: Role of lipid emulsions in cholestasis associated with long-term parenteral nutrition in children. J Parenter Enteral Nutr 2000;24:345-350.

51 Moseley RH: Sepsis-associated cholestasis. Gastroenterology 1997;112:302-305.

- 52 Lienhardt A, Rakotoambinina B, Colomb V, et al: Insulin secretion and sensitivity in children on cyclic total parenteral nutrition. J Parenter Enteral Nutr 1998;22:382-386.

53 Belli DC, Fournier LA, Lepage G, et al: Total parenteral nutrition-associated cholestasis in rats: comparison of different amino acid mixtures. J Parenter Enteral Nutr 1987;11:6773.

54 Goulet O, de Potter S, Antebi H, et al: Longterm efficacy and safety of a new olive oil-based intravenous fat emulsion in pediatric patients: a double-blind randomized study. Am J Clin Nutr 1999;70:338-345.

55 Clayton PT, Whitfield P, Iyer K: The role of phytosterols in the pathogenesis of liver complications of pediatric parenteral nutrition. Nutrition 1998;14:158-164.

- 56 Popinska K, Kierkus J, Lyszkowska M, et al: Aluminum contamination of parenteral nutrition additives, amino acid solutions, and lipid emulsions. Nutrition 1999;15:683-686.
Ben Hariz M, Goulet O, De Potter S, et al: Iron overload in children receiving prolonged parenteral nutrition. J Pediatr 1993;123:238241.

-58 Moukarzel A, Song MK, Buchman AL, et al: Excessive chromium intake in children receiving total parenteral nutrition. Lancet 1992; 339:385-388.

59 Ling PR, Sheikh M, Boyce P, et al: Cholecystokinin (CCK) secretion in patients with severe short bowel syndrome (SSBS). Dig Dis Sci 2001;46:859-864.

-60 Capron JP, Gineston JL, Herve MA: Metronidazole in prevention of cholestasis associated with total parenteral nutrition. Lancet 1983; 446-447.

-61 Heubi JE, Wiechmann DA, Creutzinger V, et al: Tauroursodeoxycholic acid (TUDCA) in the prevention of total parenteral nutrition-associated liver disease. J Pediatr 2002;141:237242.

62 Bresson JL, Narcy P, Putet G, et al: Energy substrate utilization in infants receiving total parenteral nutrition with different glucose to fat ratios. Pediatr Res 1989;25:645-648.

63 Kaufman SS: Prevention of parenteral nutrition-associated liver disease in children. Pediatr Transplant 2002;6:37-42.

64 MacFie J: Cyclic parenteral nutrition. Nutrition 1997;13:46-48.

65 Advenier E, Landry C, Colomb V, et al: Aluminum contamination of parenteral nutrition and aluminum loading in children on longterm parenteral nutrition. J Pediatr Gastroenterol Nutr 2003;36:448-453.

66 Colomb V, Goulet O, Ricour C: Home enteral and parenteral nutrition. Baillieres Clin Gastroenterol 1998;122:877-894.

67 Scolapio JS: A review of the trends in the use of enteral and parenteral nutrition support. $\mathbf{J}$ Clin Gastroenterol 2004;38:403-407.

68 Kaufman S, Atkinson JB, Bianchi A, Goulet O, Grant D, Langnas A, et al: Indications for pediatric intestinal transplantation: A position paper of the American Society of Transplantation. Pediatr Transplant 2001;5:80-87.
69 Colomb V, Jobert-Giraud A, Lacaille F, Goulet O, Fournet JC, Ricour C: Role of lipid emulsions in cholestasis associated with long-term parenteral nutrition in children. JPEN J Parenter Enteral Nutr 2000;24:345-350.

70 Iyer K, Horslen S, Torres C, et al: Histology is not predictive of functional liver recovery in parenteral-nutrition associated liver dysfunction (abstract). Pediatr Transplant 2003;7:69.

71 Bueno J, Ohwada S, Kocoshis S, et al: Factors impacting the survival of children with intestinal failure referred for intestinal transplantation. J Pediatr Surg 1999;34:27-33.

72 Fryer J, Pellar S, Ormond D, Koffron A, Abecassis M: Mortality in candidates waiting for combined liver-intestine transplants exceeds that for other candidates waiting for liver transplants. Liver Transplant 2003;9:748753.

73 Grant D, Abu-Elmagd K, Reyes J, Tzakis A, Langnas A, Fishbein T, Goulet O: Farmer D, on behalf of the Intestine Transplant Registry: 2003 report of the intestine transplant registry: a new era has dawned. Ann Surg 2005;241: 607-613.

74 Beath SV, Booth IW, Murphy MS, et al: Nutritional care and candidates for small bowel transplantation. Arch Dis Child 1995;73:348350

75 Fishbein TM, Schiano T, LeLeiko N, et al: An integrated approach to intestinal failure. Results of a new program with total parenteral nutrition, bowel rehabilitation, and transplantation. J Gastrointest Surg 2002;6:554-562.

76 Guarino A, De Marco G, Italian National Network for Pediatric Intestinal Failure: Natural history of intestinal failure, investigated through a national network-based approach. J Pediatr Gastroenterol Nutr 2003;37:136141

77 Kocoshis SA, Beath S, Booth IW, Oliva GA, Goulet O, Kaufman SS, Lai HS, Luque C, Ohtsuka Y: Intestinal failure and small bowel transplantation, including clinical nutrition: Working Group report of the second World Congress of Pediatric Gastroenterology, Hepatology and Nutrition. J Pediatr Gastroenterol Nutr 2004;39(suppl 2):S655-S661. 